\title{
DOCUMENTATION
}

\section{Les chemins de fer transpyrénéens}

Le 18 juillet 1928, Sa Majesté le roi d'Espagne et M. Doumergue, président de la République française, inauguraient la ligne de Bedous (Basses-Pyrénées), à Jaca (Espagne), première traversée de la châne des Pyrénées.

Un an plus tard, le 21 juillet 1929 , ćtait inaugurée la deuxième

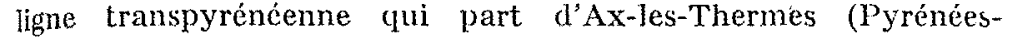
Orientales) pour aboutir à Puigcerda (Espagne).

Par suite de son tracé, cette ligne comporte de nombreux ouvrages d'art, dont le tunnel hélicoidal de Saillens (1.750 mètres) et le tunnel de Puymorens (5.355 mètres), où se trouve le point culminant de la ligne ( 1.567 metres d'altitude). Les pentes sont de l'ordre de $40 \mathrm{~mm}$. par mètre.

En Espagne, la ligne se continue par celle de Puigcerda à Rippoll, où l'on traverse le tunnel de Tosas (3.850 mètres), dont la construction, par suite des terrains très hétérogènes qui y ont été rencontrés, présenta des difficultés telles, qu'on envisagea même un autre trace.

Les trains ont un tomnage de 300 tonnes pour les express et de 360 tonnes pour les trains de marchandises. Ils sont remorqués pa: des loconiotives B. B. des Chemins de fer du Midi, alimentés a 1.500 volts, courant continu.

Le courant provient du poste le Portet-St-Simon, près de Tonlouse. La ligne à 60.000 volts entre Portet-St-Simon et Foix (70 km.) est constituce par trois conducteurs d'aluminium de $172 \mathrm{~mm}^{2}$, et est protégée par un câble de terre de $60 \mathrm{~mm}^{2}$. Entre Foix et Ax-les-Thermes $(50 \mathrm{~km}$.), la ligne est constituée par trois conducteurs d'almélec de $182 \mathrm{~mm}^{2}$. Les pylônes sont du type 150.000 volts des Chemins de fer du Midi, avec portée de $250 \mathrm{~m}$ Entre Ax-les-Thermes et Latour-de-Carol, la ligne est constituce

\section{Ligne d'Ax-les-Termes à Puigcerda}

par trols conducteurs de cuivre de $53,7 \mathrm{~mm}^{2}$. Sur le parcours l'Hospitalet-Portat, c'est-à-dire pour la traversée du cál de Puymorens, la ligne est dédoublée, pour éviter une interruption de courant, provoquée par une avalanche.

La ligne caténaire est du type normal des Chemins de fer du Midi, mais avec câble porteur en bronze de $140 \mathrm{~mm}^{2}$.

Les sous-stations sont placées à Ax-les-Thermes, l'Hospitalet, et Latour-de-Carol, distantes de 20 et $18 \mathrm{~km}$. Elles sont toutes semblables, et comprennent deux groupes de $2.000 \mathrm{KW}$., composés chacun d'un transformateur $60.000 / 2 \times 595$ volts à deux enroulements secondaires et deux commutatrices héxaphasées en série de $1.000 \mathrm{KW}$ à 750 volts. Celles-ci sont protégées par deux disjoncteurs ultra-rapides dont un sur le circuit de terre qui, en cas de court-circuit, inserre une résistance limitative et coupe la bobine de maintien du disjoncteur ultra-rapide, côté ligne.

Les batteries d'accumulateurs sont du type cadmium-nicke à 102 éléments, dont 40 éléments de réduction. Leur capacité est de 88 ampères-heures, au régime de decharge de 5 heures.

Le poste de transformation de Latour-de-Carol comporte, en plus, deux transformateurs $60.000 / 10.000$ volts de $250 \mathrm{KVA}$., destinés aux services auxiliaires de force et d'éclairage de la gare.

L'amélioration du trafic ferroviaire France-Espagne sera encore plus sensible à la fin de 1930 , lorsque la voie espagnole sera ramenée à l'écartement normal jusqu'à Barcelone, ainsi que l'a décidè le gouvernement espagnol.

Le Génie civil, 3 août 1929.

\section{L'usine hydro-électrique de la Bonne-Inférieure de la Société des Forces motrices Bonne-et-Drac}

L'anćnagement de la chute de la Bonne-Inférieure, commencé an 1917 par le groupe du Bon Drac, puis interrompu par suite des difficultés économiques d'après guerre, a été repris à la fin de 1923 par la Société Forces motrices Bonne et Drac. Elle a été mise en service en 1926 .

Construite sur la rive droite du Drac, un peu en amont de son confluent avec la Bonne, l'usine génératrice a des dispositions assez particulières, imposées par la topographie des lieux. En effet, le Drac coulant au fond d'une gorge très encaissée, on a cherché à réduire au minimum la largeur du bâtiment, afin d'éviter d'importants terrassements. D'autre part, il était indispensable de placer le sol des machines à une cote assez élevée pour être à l'abri des crtes et aussi pour permettre ultérieurement la création d'un réservoir de compensation. Ces diverses conditions ont conduit à adopter des groupes à axe vertical et à construire une partie du batiment en encorbellement.

La puissance installće est cle 6.100 KVA, répartie en deux alternateurs de $3.050 \mathrm{KVA}$, accouplés à des turbines centripètes de 3.500 chevaux, fonctionnant sous 70 mètres de chute. Les alter- nateurs ont une tension normale de 5.250 volts. Deux transformateurs triphasés de 1.500 KVA, $5.250 / 10.500$ volts alimentent un départ à 10.500 volts.

A 200 mètres de l'usine, et séparé d'elle par un éperon rocheux, se trouve un poste de transformation extérieur à 50.000 volts, comprenant trois transformateurs triphasés de 2.000 KVA, $5.250 / 28.800$ volts avec le couplage triangle-triangle, ou 5.250 $/ 50.000$ volts avec le couplage triangle-étoile. L'exiguité du terrain a obligé à donner à ce poste un faible encombrement en surface et à le développer principalement dans le sens de la hauteur.

La Socicté Forces motrices Bonne et Drac a confié l'exploitation de l'usine de la Bonne-Inférieure d̀ la Société hydro-électrique de Fure et Morge et de Vizille, qui utilise actuellement sur son réseau de distribution toute l'energic produite. L'usine assurera, en outre, l'alimentation du chemin de fer de La Mure à Gap, dont les travaux sont en cours.

André RoBsnT. - R.G.L., 26 oct. 1929).

\section{Les transporteurs aériens du barrage de Grimsel}

Situé à 1.850 motres d'altitude, dans la région de l'Oberhasli, le lac de Grimsel est peu important, mais il peut etre facllement transformé en un réservoir de 100 millions de mètres cubes au moyen d'un barrage de $\mathbf{1 1 0}$ mètres de hauteur. Son aménagement en a ćté entrepris par la Société des Forces motrices bernoises. Par suite de sa situation géographique, la coulée du béton ne peut se faire que pendant trois mois de l'année.

La fabrication du béton se fait sur une hauteur voisine, sur la rive droite de l'Aare. Trois trémies le distribuent aux bernes in transporteur. De la gare d'Imnertkirchen au chantier, le transport des matériaux se fait au moyen d'un transporteur à câble de $18 \mathrm{~km}$. de longueur ayant un débil jourmalier de 200 tomnes.

par suite de la largeur du barage de 75 mètres, il a fallu installer deux transporteurs à càbles parallèles pour l'alimentation des dispositifs de coulée. Ceux-ci sont constitués par des poutrelles triangulaires en treillis et ont une portée de 23 mètres. La partie inférieure se termine par une goulette de 200 millimètres de diamètre en dessous de laquelle peut se déplacer un bras de 10 métres de long.

En plus de la poutrelle de coulée, chaque transporteur est muni d'une plateforme en trelllis d'une longueur de 35 mètres, elles sont destinées à exécuter les travaux de revêtement. Chaque transporteur a un débit horaire de 78 mètres cubes de béton. L'ensemble permet donc de couler 2.000 métres ctubes. Le coût de l'installation dépasse 2.000 .000 de franes.

Zeitschrift des Vereines Dentscher Ingenieure, 30 mars 1929. 


\section{Déversoir de crues sur le Mississipi près de Cairo (U. S. A.)}

Le Mississipi est sujet à de très fortes crues qui ont obligé l'ctablissement de digues importantes. En particulier la ville de Cairo est protégée par une digue permettant des crues de $18 \mathrm{~m} .30$, correspondant à un débit de $68.000 \mathrm{~m}^{\mathrm{S}}$ par seconde. On a déjà enregistré des crues de $18 \mathrm{~m}$. En cas de rupture de la digne, ce serait la submersion totale de la ville.

Aussi, a-t-on décidé de créer un réservoir par l'abaissement de $1 \mathrm{~m} .50$ de la crête de la digue, sur une longueur de $18,5 \mathrm{~km}$., en aval de la ville de Cairo, et un autre de $8 \mathrm{~km}$. de longueur en amont de la ville de New-Madrid, à $56 \mathrm{~km}$. de Cairo en ligne droite. Une digue à peu près rectiligne reliera ces deux villes. On aura ainsi une zone sacrilice de 53.000 hectares, où l'eau se déversera lorsque la crue atteindra $16 \mathrm{~m}$. 77. Le calcul de ce déversoir a montré qu'on abaisserait de $0 \mathrm{~m} .30$ au droil de Cairo le niveau des crues de $68.000 \mathrm{~m}^{3} /$ seconte.

Ce projet est trés disculi parce que, après les crues, il se produit une vigetation spontance abondante, qui diminuera rapidement le clébit calculé. De plus, les dégàts à la propriété seront tels cue cela entrainera l'abandon de la région par les fermiers. C'est done une prime l'assurance bien chère que représentent les frais d'exécution du projet et de dédommagement. Le Géne civil, 26 octobre 1929.

\section{La production de l'électricité en Hollande}

La production de l'électricité en Hollande a subi un développement remarquable durant ces dernières années. La consommation moyenne est de $145 \mathrm{KW}$ heures par habitant. II faut voir, parmi les causes de cet accroissement rapide de la consommation, le fait que le tarif de l'énergie industrielle est de 0 fr. 20 le kilowatt heure, celui des usages domestiques de $0 \mathrm{fr}$. 40 , ramené à $0 \mathrm{fr} .20$ pendant la muit. Liabomé paie the ammilé fixe au tarif éclairage et le reste utilisé dans les réchauds, fours, etc., marqué par un compteur à double tarif, (un journalier, l'autre nocturne), all prix de l'énergie à usage domestique.

Elektrotechnische Zeitschrit, 13 juin 1929.

\section{Centrale hydraulique en plein air}

La centrale de Norwood, construite par la "Carolina Power and Light $\mathrm{C}^{\circ}$ " a ceci de particulier qu'en dehors de la salle de manouvre qui est dans un bâtiment, tout le reste est en plein air. La centrale comprend une génératrice de $18.000 \mathrm{KW}$. et deux génératrices de $22.000 \mathrm{KW}$. Les turbines sont à axe vertical avec alternateurs au-dessus; ceux-ci sont protégés au moyen diun carter en tole. Un pont roulant de 150 lomes sert it la manutention. Il roule sur deux rails places l'un sur le barrage et l'autre sur le sol. Le poste de transformation qui lui est adjacent est également à l'air libre.
L. T. Z, is juil. 1929 .

\section{Emploi de l'électricité dans la}

L'Indiana Limestone Company de Bedford (Indiana) fut constituée en 1926 par la réunion de vingt-quatre carrières et manufactures de pierres à bâtir. Devant la demande considérable, principalement en pierres calcaires, cette Société a organisé d'une façon tout à fait moderne ses carrières et ses ateliers. La force électrique a été largement employée pour actionner les scies à ruban, les scies circulaires, les tours, les raboteuses, les polisseuses, les broyeuses, les compresseurs actionnant les marteaux et burins pneumatiques pour la sculpture des corniches et des chapiteaux. Il est intéressant de noter que le calcaire s'usine d'une manière

\section{production des pierres à bâtir}

semblable à lacier ou à la fonte.

L'Indiana Limestone Company foumit 65 or des pierres a bâtir usinces, utilisées aux Etats-Unis, comprenant toutes sortes cle variétés : granit, calcaire, grès, marbre. Elle utilise une puissance globale de $20.500 \mathrm{KVA}$. répartie en cent groupes de transformation. La tension dutilisation est de 220 volts, courant triphasé 60 périodes. L'énergie provient des lignes de transport à 33.000 et 66.000 volts, ainsi que des réseaux a 4.100 volts.

General Electric Review, août 1929.

\section{INFORMATIONS}

\section{Sur la route d'hiver des Alpes en autocar P.-L.-M.}

Les services automobiles de la Route d'Hiver des Alpes fonctionnent entre Nice et Aix-les-Bains Mont Revard. Deux départs par semaine dans chaque sens : jeudi et dimanche de Nice; mercredi et dimanche d'Aix-les-Bains.

Jusqu'au 20 décembre, un troisième service fonctionne entre Nice et Grenoble : départ cle Nice le mardi, de Grenoble le samedi. Le parcours de Nice à Aix-les-Bains s'effectue en trois jours par Entrevaux, Annot, Digne, Sisteron, le col de la Croix-Haute $(1.176 \mathrm{~m}$.), Grenoble, le col de la Porte $(1.354 \mathrm{~m}$.) et Saint-Pierre-deChartreuse ou vice versa.

Du 21 décembre au 28 février, les services sont prolongés d'Aixles-Bains sur, Chamonix afin de relier entre elles les grandes stations des sports d'hiver : Chamonix, Combloux, Mégère, Aix-lesBains-Mont-Revard, Saint-Pierre-de-Chartreuse; ils ont lieu trois fois par semaine dans chaque sens du 21 decembre au 31 janvier, tous les jours du $1^{\text {er }}$ au 28 février.

Du $1^{\text {er }}$ mars au 20 mai, les services continuent à fonctionner chaque jour, mais ils n'ont lieu qu'entre Nice et Aix-les-Bains.

\section{Le Maroc par Marseille}

Pour aller de France au Maroc, embarquez à Marseille. C'est la traversée la plus rapide. C'est la plus économique.
Les confortables navires de la compagnie paquet partent de Marseille tous les samedis à destination de Tanger et de Casablanca.

Dans le sens Maroc-France, les départs ont lieu pour Marseille :

De Casablanca : tous les samedis à 16 heures.

De Tanger : tous les dinanches.

Dans le sens France-Maroc, les départs ont lieu :

De Marseille : tous les samedis a 11 heures.

Le prix du passage comporte la nourriture à bord, vin compris. Les voyageurs n'ont pas à quitter le parjuebat pour emprunter d'autres moyens de transport nécessitant des frais d'hôtel.

Des billets simples, valables quinze jours, et des billets d'aller et retour valables trente et quatre-vingt-dix jours, domnant droit à J'enregistrement direct des bagages, sont dolivrés pour Tanger et Casablanca dans les principales gares du P, L,$-M$. et dans les agences de la Compagnie Paquet.

De plus, les voyageurs qui présentent un titre de parcours à destination de marseille, un avis de la Compagnie Paquet (lettre ou télćgramme) constatant que leurs places sont bien retenues sur le paquebot, peuvent faire enregistrer leurs bagages directement pour Tanger ou Casablanca, en s'affranchissant ainsi des emnuis résultant soil du relrail des bagages, soil du transit do ceux-ci à Marseille. 